\section{Active Telephonic Follow-up During COVID-19 Lockdown: Initial Experience}

The severe acute respiratory syndrome coronavirus-2 (SARSCoV-2) pandemic forced a lockdown in India in late March, 2020. Prior to this, our department was working normally with 30-35 pediatric surgeries per week. As the lockdown was announced, a lot of patients who were discharged recently were lost to follow-up in the immediate post-operative period. We herein present our initial experience with telephonic follow-up and management of these children.

We prospectively collected the details of telephonic follow-of pediatric surgical patients discharged in the preceding two weeks period (10-24 March, 2020) before the nationwide lockdown. Data of children with day-care procedures was not collected. Parents of children eligible for study were called on the mobile numbers provided at the time of admission. In case of failure to contact, it was decided to stop calling after three days of twice daily calls. The problems identified, advice given and the outcome were recorded.

A total of 32 children were discharged during this period, out of which 26 families $(81.2 \%)$ could be contacted. Of these, $10(38.5 \%)$ had already paid a visit in the postoperative period and did not require any intervention, whereas the remaining had not visited since discharge and were not under follow-up. Seven children $(26.9 \%)$ fared well in the post-operative period and were advised stitch removal from local health center. Nine (34.6\% of contactable patients) parents had complaints viz, ostomy diarrhea with dehydration in three neonates (responded well to intravenous fluid administration with the help of local medical practitioner); two children with urethral stent in situ (stent was removed by telephonic coordination with local practitioner following which the patients did well), and one neonate with Peri-gastrostomy tube leakage and dehydration (managed successfully with telephonic advice for gastrostomy tube removal at nearby Primary Health Centre). However, three patients were advised to come to the hospital. One patient having in situ DJ stent for seven weeks with symptoms of pyelonephritis was managed by DJ stent removal, and a patient of adhesive obstruction was successfully managed conservatively and discharged after 6 days. The third child with slipped feeding jejunostomy was re-operated. Exploratory laparotomy with T-tube closure of perforation was performed but the child died on day 12 due to septicemia.

Postoperative follow-up of pediatric general surgical patient is important for ensuring and maintaining optimal patient outcomes [1], as complications in the early postoperative period have high morbidity and mortality [2]. Although there was no movement restriction for patients during the lockdown, still due to non-availability of public transport, travel was difficult for most patients [3].
Communication on phone can be an effective option for consultation during follow-up [4]. In the present study, we received good response both from the parents and the locally available doctors. Even in this era of telecommunication, with a lot of people having a mobile phone, surprisingly none of the patients with problems contacted us. This may be because patients do not feel comfortable in contacting the treating doctor on phone, and probably need to be proactively informed about the availability of this mode for problem resolution.

Most parents informed that initially the local general practitioners, when contacted, were not willing to handle these patients due to the notion of complication in a post-operative child and apparent lack of skills to treat them. With telephonic contact with the local general practitioners, we were able to manage 6 out of 9 complications $(66.6 \%)$ with their help. Though there are chances of miscommunication on phone and difficulty in interpretation of all the instruction, one can avoid it if standard operating procedures are developed and followed diligently [5].

We propose from our experience that in wake of any event affecting the movement of patients, active efforts and call from the treating doctors can make a lot of difference with minimal effort and energy. Recent guidelines on telemedicine will go a long way in strengthening this model of patient care [6].

Published online: June 24, 2020; PII S097475591600202

\begin{tabular}{|c|}
\hline 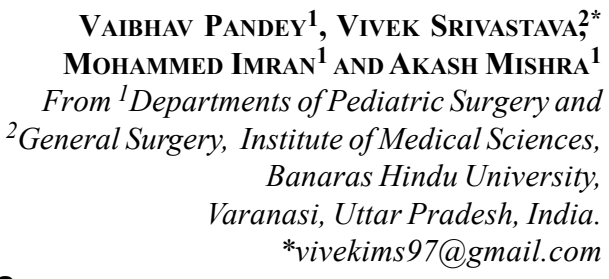 \\
\hline
\end{tabular}

\section{REFERENCES}

1. Pawar D. Common post-operative complications in children. Indian J Anaesth. 2012;56:496-501.

2. Attard TM, Miller M, Walker AA, Lee B, McGuire SR, Thomson M. Pediatric elective therapeutic procedure complications: A multicenter cohort analysis. J Gastroenterol Hepatol. 2019;34:1533-9.

3. Paital B, Das K, Parida SK. Inter nation social lockdown versus medical care against COVID-19, a mild environmental insight with special reference to India. Sci Total Environ [Internet]. 2020;728:138914.

4. Fischer K, Hogan V, Jager A, Allmen D von. Efficacy and utility of phone call follow-up after pediatric general surgery versus traditional clinic follow-up. Perm J. 2015;19:11.

5. Reisman AB, Brown KE. Preventing communication errors in telephone medicine: A case-based approach. J Gen Internal Med. 2005;20:959-63.

6. Mahajan V, Singh T, Azad C. Using telemedicine during the COVID-19 pandemic. Indian Pediatr. 2020 May 14;S097475591600182 [Online ahead of print]. 